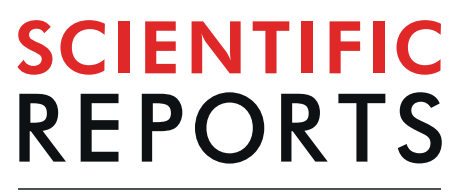

natureresearch

\title{
Peripapillary microvascular changes in patients with systemic hypertension: An optical coherence tomography angiography study
}

Yong-II Shin ${ }^{1}$, Ki-Yup Nam ${ }^{2}$, Woo-Hyuk Lee ${ }^{1}$, Cheon-Kuk Ryu ${ }^{1}$, Hyung-Bin Lim ${ }^{1}$, Young-Joon Jo ${ }^{1}$ \& Jung-Yeul Kim (ib) ${ }^{1 *}$

The purpose of this study was to investigate changes in peripapillary microvasculature using optical coherence tomography angiography (OCTA) in systemic hypertension (HTN) patients. This was a crosssectional study. Based on the duration of HTN, seventy-eight $\mathrm{HTN}$ patients were divided into two groups. (HTN group 1: $<10$ years, 38 eyes; HTN group 2: $\geq 10$ years, 40 eyes) and 90 control subjects. All subjects underwent $6 \times 6 \mathrm{~mm}$ OCTA scan centered on the optic nerve head. We analyzed peripapillary vessel density (VD) and perfusion density (PD) in superficial capillary plexus among three groups. The average ganglion cell-inner plexiform layer (GC-IPL) and retinal nerve fiber layer (RNFL) thicknesses of HTN group 2 were thinner than those of the control group $(p=0.016$, and 0.035 , respectively). HTN group 2 showed lower peripapillary VD and PD than the control group. However, there were no differences between HTN group 1 and the control group in OCT and peripapillary OCTA parameters. In HTN patients, the peripapillary VD, PD and GC-IPL, RNFL thicknesses correlated significantly. OCTA showed that the peripapillary VD and PD were lower in HTN patients with a duration $\geq 10$ years compared with those of normal controls. Peripapillary microvasculature was correlated with the RNFL and GC-IPL thicknesses. HTN duration should therefore be considered when evaluating peripapillary microvasculature using OCTA.

Cardiovascular disease is one of the more common causes of death in developing country, and hypertension (HTN) is the most common treatable risk factor. According to a US study, approximately 68 million (31\%) of patients $\geq 18$ years of age are reported to have $\mathrm{HTN}^{1}$, and approximately one billion worldwide are affected by $\mathrm{HTN}^{2}$. With advances in medical technology, the life expectancy has been extended, and the number of patients with HTN has increased.

Uncontrolled HTN can cause vascular changes in many organ systems, such as the brain, heart, kidneys, and eyes, due to elevated arterial pressure and increased peripheral resistance. Vascular changes in the eye can be directly visualized using funduscopy. Arteriolar narrowing is a hallmark of hypertensive retinopathy (HTNR), and the narrowing being characterized as either focal or diffuse. This occurs when the systemic blood pressure rises to maintain constant blood flow by autoregulation of the retinal circulation. The destruction of the inner blood-retinal barrier and vascular endothelial structure can then lead to cotton wool spots, retinal hemorrhage, and intraretinal lipid deposits.

Funduscopy, optical coherence tomography (OCT), and fluorescein angiography (FA) may be helpful in the diagnosis and evaluation of retinal pathological changes in HTN. In our previous OCT study, the ganglion cell-inner plexiform layer (GC-IPL) and peripapillary retinal nerve fiber layer (RNFL) thicknesses were lower in chronic HTN and relieved HTNR patients than normal controls ${ }^{3,4}$, Furthermore, in previous optical coherence tomography angiography (OCTA) studies, foveal microvascular perfusion was decreased, which correlated with GC-IPL thinning in patients with $\mathrm{HTN}^{5,6}$.

${ }^{1}$ Department of Ophthalmology, Chungnam National University College of Medicine, Daejeon, Republic of Korea. ${ }^{2}$ Department of Ophthalmology, Gyeongsang National University Changwon Hospital, Changwon, Republic of Korea.*email: kimjy@cnu.ac.kr 
We assumed that RNFL thinning in patients with HTN should be related with peripapillary microvascular changes. We therefore analyzed changes in peripapillary microvasculature in patients with HTN using OCTA, and identified the associations between OCT and peripapillary OCTA parameters.

\section{Methods}

Subjects. This retrospective, cross-sectional study was approved by the institutional review board of Chungnam National University Hospital, and was conducted to the tenets of the Declaration of Helsinki. The requirement for obtaining informed patient consent was waived by the institutional review board of Chungnam National University Hospital due to the retrospective nature of the study. The medical records at the Chungnam National University Hospital inclusive were reviewed to identify HTN patients who visited retina clinic.

Diabetic patients were excluded because diabetes significantly affects the inner retinal layer and microvasculature. HTN was diagnosed as systolic blood pressure $\geq 140 \mathrm{mmHg}$ or diastolic blood pressure $\geq 90 \mathrm{mmHg}^{7}$ at Chungnam National University Hospital. They underwent regular follow-up for blood pressure control, and all participants were well-controlled blood pressure for at least the past year. The participants were divided into three groups: control group (negative history of systemic disease and no ocular disease, 90 eyes), HTN group 1 (patients with $\mathrm{HTN}<10$ years, 38 eyes), and HTN group 2 (patients with $\mathrm{HTN} \geq 10$ years, 40 eyes). We excluded HTNR using fundus photography of patients in the HTN groups. One eye from each participant was included. Only one eye was randomly selected if both eyes were eligible. In the power calculation using $\mathrm{G}^{*}$ Power $3.1^{8}$, the power of the average peripapillary VD and PD were 0.970 and 0.967 , respectively.

The exclusion criteria were systemic diseases other than HTN and hyperlipidemia, glaucoma, optic nerve disorders, a best-corrected visual acuity $(\mathrm{BCVA})<20 / 25$, intraocular pressure (IOP) $>21 \mathrm{mmHg}$, high myopia [axial length $(\mathrm{AL}) \geq 26.0 \mathrm{~mm}$ or spherical equivalent $(\mathrm{SE})>-6$ diopters], and a history of intraocular surgery.

All patients underwent slit-lamp biomicroscopy, BCVA, SE, IOP, and AL using an IOL master, fundus photography, dilated fundus examination, OCT, and OCTA.

Optical coherence tomography (OCT) and optical coherence tomography angiography (OCTA) measurement. OCT examination was performed using a spectral domain OCT (Cirrus HD-OCT; Carl Zeiss Meditec, Dublin, CA, USA). The skilled examiner performed all imaging. Central macular thickness (CMT) and GC-IPL thickness were analyzed using a macular cube scan. Average RNFL thickness was analyzed using an optic disc cube scan. We excluded with signal strength (SS) less than 7 or poor centration or segmentation error in OCT scans.

OCTA imaging was acquired using the Zeiss Cirrus HD-OCT with Angioplex, with a light source at $840 \mathrm{~nm}$ and an A-scan rate of $68 \mathrm{KHz}$. We used only scans without segmentation errors and motion artifacts and with SS greater than 9 . The superficial capillary plexus (SCP) images were segmented with an internal limiting membrane and the inner plexiform layer and the deep capillary plexus (DCP) was segmented with an inner nuclear layer and the outer plexiform layer.

The optic disc centered $6 \times 6 \mathrm{~mm}$ OCTA scans were acquired to evaluate peripapillary microvasculature. A $6 \times 6 \mathrm{~mm}$ scans were acquired containing $350 \times 350 \mathrm{scans}$ each, and B-scans were repeated twice at each cross-section. Vessel density (VD) and perfusion density (PD) are defined as follows; VD means total length of perfused vasculature per unit area in a region of measurement and PD means the total area of perfused vasculature per unit area in a region of measurement. The AngioPlex software automatically displays the measured value of the SCP according to the subfield of the ETDRS. We only studied SCP because the built-in software provided automatically quantified values of SCP only. We analyzed the peripapillary VD and PD of the average and quadrants of the inner ring, outer ring, and full area (Fig. 1). We included with SS more than 9 and without segmentation errors and motion artifacts in OCTA images.

Statistical analysis. Statistical analysis were performed with SPSS version 22.0 (Chicago, IL, USA). For statistical analyses, BCVA values were transformed to the logarithm of the minimum angle of resolution (log MAR) values. One-way ANOVA with Bonferroni's post-hoc test and the chi-squared test were applied to compare the clinical characteristics, OCT, and OCTA parameters among groups. To determine the relationship between peripapillary OCTA and OCT parameters, Pearson's correlation was used. $\mathrm{P}<0.05$ was considered statistically significant.

\section{Results}

Patient characteristics. After excluding poor quality OCTA images (6 eyes of HTN group 1, 7 eyes of HTN group 2, and 6 eyes of normal controls), Seventy-eight eyes from HTN patients (38 in HTN group 1 and 40 in HTN group 2) and 90 control eyes were enrolled in the study. The durations of HTN were $4.2 \pm 2.4$ years in HTN group 1 and $16.3 \pm 5.8$ years in HTN group $2(p<0.001)$. There were no significant difference among the three groups in age, sex, laterality, BCVA, SE, IOP, AL, and SS of OCTA images (Table 1).

OCT measurements. The CMT did not show a difference among the three groups $(p=0.135)$. The GC-IPL and peripapillary RNFL thicknesses showed statistically significant differences in the three groups $(p=0.019$, and 0.037, respectively). Using Bonferroni's test, the GC-IPL and RNFL thicknesses in HTN group 2 (80.2 \pm 6.2 and $92.2 \pm 8.1 \mu \mathrm{m}$, respectively) were lower than those of the control group $(83.3 \pm 5.8$ and $96.0 \pm 7.7 \mu \mathrm{m}$, respectively). However, there were no differences between HTN group 1 and the control group (Table 2).

Peripapillary OCTA measurements. The mean values \pm standard deviation of OCTA measurements are listed in Table 3 (VD) and Table 4 (PD). The average peripapillary VD and PD of the inner ring, outer ring, and full area were significantly different among the three groups. Using Bonferroni's test, HTN group 2 showed a 

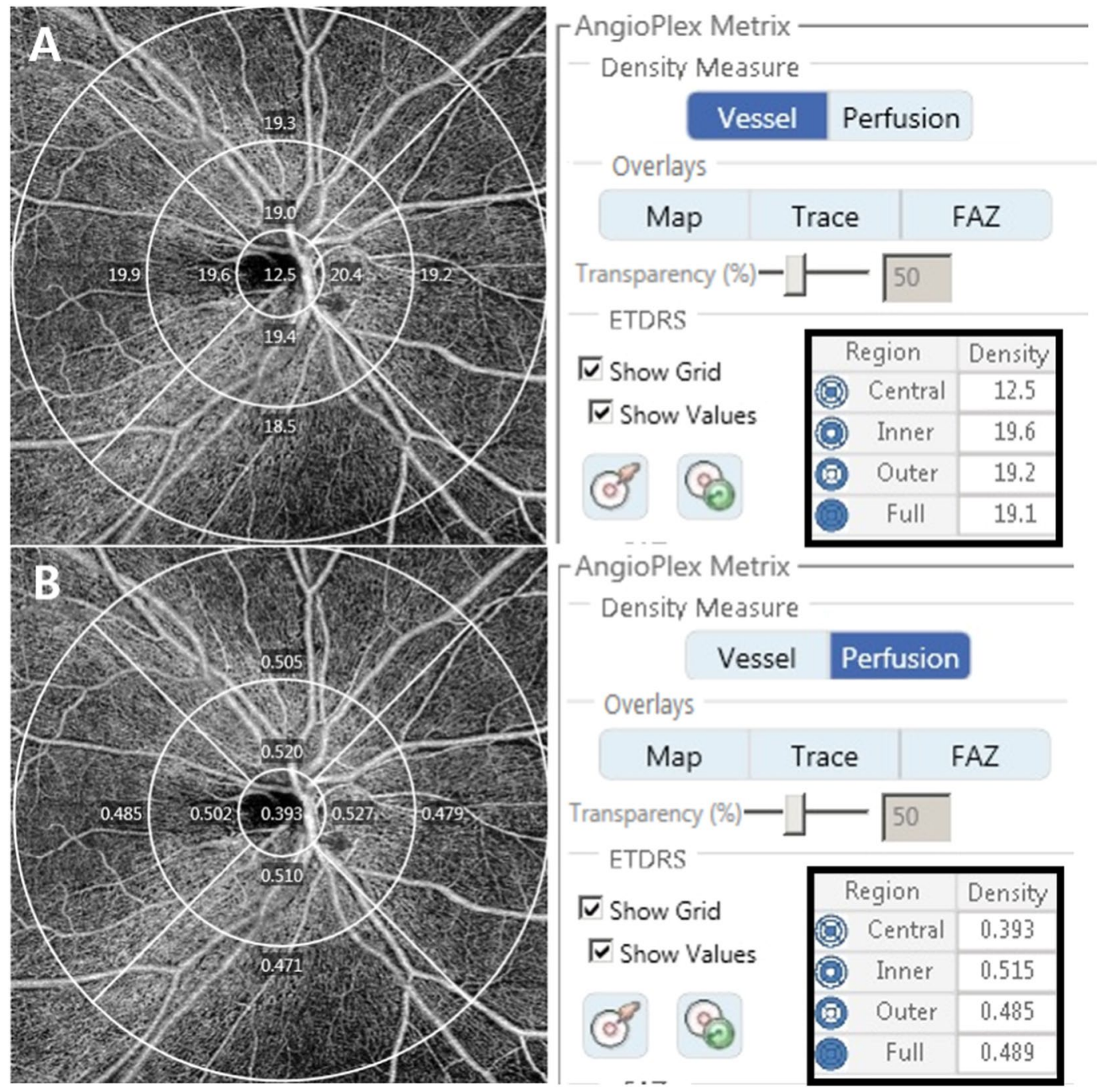

Figure 1. Optical coherence tomography angiography $6 \times 6 \mathrm{~mm} \mathrm{scan}$ image centered on the optic disc. The en face image of the superficial layer overlaid with the Early Treatment of Diabetic Retinopathy Study grid. The diameters of the three concentric circles are 1, 3, and $6 \mathrm{~mm}$. The measurement tool (AngioPlex software, version 10.0; Carl Zeiss Meditec) provided (A) peripapillary vessel density and (B) perfusion density measurements in individual subfields. The bold box shows the automatic quantitative measurements for an average of the inner ring, outer ring, and full area.

lower peripapillary VD and PD than the control group. The average of the full areas for the VD and PD and the average PD of the outer ring were lower in HTN group 2 than in HTN group 1.

Association of clinical and OCT parameters with peripapillary OCTA parameters in HTN patients. The average GC-IPL and peripapillary RNFL thicknesses were correlated with the peripapillary VD $(\mathrm{r}=0.358, p=0.001$ and $\mathrm{r}=0.239, p=0.035$, respectively $)$ and $\mathrm{PD}(\mathrm{r}=0.385, p=0.001$ and $\mathrm{r}=0.225, p=0.047$, respectively) in HTN patients (Fig. 2). The duration of HTN was not correlated with GC-IPL and RNFL thicknesses, but negatively correlated with the peripapillary VD and PD $(r=-0.219, p=0.049$ and $\mathrm{r}=-0.240$, $p=0.035$, respectively). However, age, female sex, BCVA, SE, IOP, AL, mean arterial pressure and CMT were not correlated with the average peripapillary VD and PD. Multivariate regression analysis showed that clinical factors such as age, IOP, sex, BCVA, SE, and mean arterial pressure were not significant factors affecting the average peripapillary VD and PD (see Supplementary Table 1). Age, axial length, and signal strength showed no statistically significant correlation with the average peripapillary VD and PD in normal controls.

\section{Discussion}

Systemic HTN affects the structure and function of blood vessels, and HTN changes are systemic risk factors for cardiovascular disease $^{9,10}$, stroke ${ }^{11}$, and chronic kidney disease ${ }^{12,13}$. In eyes, HTN aggravates diabetic retinopathy ${ }^{14}$, increases the risk of retinal vascular occlusive disease $e^{15,16}$, and is associated with ischemic optic neuropathy. ${ }^{17}$ In addition, HTN is associated with ocular diseases such as progression of glaucoma ${ }^{18,19}$, and increases in choroidal neovascularization (CNV) in the fellow eye of patients with one eye affected with $\mathrm{CNV}^{20}$. 


\begin{tabular}{|l|l|l|l|l|}
\hline & $\begin{array}{l}\text { Control group } \\
(\mathbf{n = 9 0 )}\end{array}$ & $\begin{array}{l}\text { HTN group 1 } \\
(\mathbf{n}=\mathbf{3 8})\end{array}$ & $\begin{array}{l}\text { HTN group 2 } \\
\mathbf{( n = 4 0 )}\end{array}$ & p-value \\
\hline Age (years) & $60.1 \pm 8.9$ & $60.6 \pm 10.1$ & $62.8 \pm 8.4$ & $0.292^{*}$ \\
\hline Sex (male/female) & $38 / 52$ & $22 / 16$ & $15 / 25$ & $0.154^{\dagger}$ \\
\hline Laterality (od/os) & $44 / 46$ & $17 / 21$ & $20 / 20$ & $0.882^{\dagger}$ \\
\hline Duration of hypertension (years) & & $4.2 \pm 2.4$ & $16.3 \pm 5.8$ & $<\mathbf{0 . 0 0 1}^{\ddagger}$ \\
\hline Systolic blood pressure (mmHg) & $115.6 \pm 9.7$ & $119.1 \pm 8.3$ & $117.1 \pm 8.8$ & $0.148^{*}$ \\
\hline Diastolic blood pressure (mmHg) & $79.8 \pm 7.6$ & $82.2 \pm 5.3$ & $81.1 \pm 6.3$ & $0.182^{*}$ \\
\hline BCVA (log MAR) & $-0.02 \pm 0.08$ & $-0.01 \pm 0.07$ & $0.02 \pm 0.08$ & $0.123^{*}$ \\
\hline Spherical equivalent (diopters) & $-0.39 \pm 2.06$ & $0.07 \pm 1.24$ & $0.01 \pm 1.43$ & $0.289^{*}$ \\
\hline Intraocular pressure (mmHg) & $14.3 \pm 2.9$ & $15.2 \pm 2.6$ & $15.2 \pm 3.0$ & $0.118^{*}$ \\
\hline Axial length (mm) & $23.71 \pm 0.96$ & $23.85 \pm 0.84$ & $23.62 \pm 0.89$ & $0.542^{*}$ \\
\hline Signal strength & $9.7 \pm 0.5$ & $9.6 \pm 0.5$ & $9.6 \pm 0.5$ & $0.571^{*}$ \\
\hline
\end{tabular}

Table 1. Demographics and clinical features of the study subjects. HTN=hypertension; BCVA = bestcorrected visual acuity; log MAR = logarithm of the minimum angle of resolution. Values are presented as mean \pm standard deviation unless otherwise indicated. ${ }^{*}$-value for one-way analysis of variance. ${ }^{\dagger} p$-value for chi-square test. ${ }^{*}$ p-value for Student's $t$-test (HTN group 1 vs. 2).

\begin{tabular}{|l|l|l|l|l|l|l|l|}
\hline & $\begin{array}{l}\text { Control group } \\
(\mathbf{n = 9 0})\end{array}$ & $\begin{array}{l}\text { HTN group } \\
\mathbf{1}(\mathbf{n}=\mathbf{3 8})\end{array}$ & $\begin{array}{l}\text { HTN group } \\
\mathbf{2}(\mathbf{n}=\mathbf{4 0})\end{array}$ & $\mathbf{p}_{\text {-value }}^{*}$ & p-value & p-value & p-value $^{\S}$ \\
\hline $\begin{array}{l}\text { Central macular } \\
\text { thickness }(\mu \mathrm{m})\end{array}$ & $251.7 \pm 18.4$ & $249.0 \pm 23.5$ & $244.0 \pm 20.7$ & 0.135 & & & \\
\hline $\begin{array}{l}\text { Average GC-IPL } \\
\text { thickness }(\mu \mathrm{m})\end{array}$ & $83.3 \pm 5.8$ & $81.9 \pm 5.2$ & $80.2 \pm 6.2$ & $\mathbf{0 . 0 1 9}$ & 0.633 & $\mathbf{0 . 0 1 6}$ & 0.588 \\
\hline $\begin{array}{l}\text { Average RNFL } \\
\text { thickness }(\mu \mathrm{m})\end{array}$ & $96.0 \pm 7.7$ & $94.1 \pm 8.5$ & $92.2 \pm 8.1$ & $\mathbf{0 . 0 3 7}$ & 0.675 & $\mathbf{0 . 0 3 5}$ & 0.824 \\
\hline
\end{tabular}

Table 2. Comparison of the central macular thickness, average GC-IPL thickness, and RNFL thickness among groups. HTN = hypertension; GC-IPL = ganglion cell-inner plexiform layer; RNFL = retinal nerve fiber layer. Values are presented as mean \pm standard deviation. ${ }^{*}$ The $p$-value was obtained using one-way analysis of variance. ${ }^{\dagger}$ The $p$-value was obtained using post hoc tests (Bonferroni) between the normal control group and HTN group 1. ${ }^{*}$ The p-value was obtained using post hoc tests (Bonferroni) between the normal control group and HTN group 2. ${ }^{\S}$ The $\mathrm{p}$-value was obtained using post hoc tests (Bonferroni) between HTN group 1 and group 2 .

HTNR is one of the markers of target organ damage in the initial evaluation of HTN patients ${ }^{21}$, and the grade of HTNR is associated with mortality ${ }^{9,22}$. thus, evaluation of retinal changes in HTN patients is clinically important.

Elevated blood pressure results in various changes to the retinal vasculature. These changes are classified as arteriolar changes (arteriolar narrowing, arteriolar wall opacification and arteriovenous nicking) and retinal lesions (retinal hemorrhage, microaneurysm, cotton wool spots, and hard exudate) caused by blood-retinal barrier damage. Hayreh reported that hypertensive changes in the eye affect not only the retina but also the choroid and optic disc ${ }^{23}$.

Funduscopy has been used to diagnose HTNR. However, if there is only arteriolar narrowing during an early stage, it may be difficult to detect these changes using only a fundus examination. Several studies have therefore been conducted to quantitate and differentiate these changes. Fundus photography has been used to measure decreases in retinal arteriolar diameter and the arteriole-to-venule ratio, and has revealed such decreases in high blood pressure patients when compared with normal subjects ${ }^{24-26}$.

OCT is widely utilized for the diagnosis and treatment in retinal diseases and glaucoma. It can distinguish ten retinal layers with high resolution, and has the advantage of quantitatively evaluating the thickness of each layer. In HTN patients without retinopathy or glaucoma, thinning of the GC-IPL and peripapillary RNFL was reported $^{3,4,27}$. In our study, there was no difference in the CMT, but the GC-IPL and RNFL thicknesses were different among the three groups, and those of HTN group 2 was thinner than the control group. In an animal study using rhesus monkeys, the RNFL thickness decreased with chronic arterial hypertension ${ }^{28}$. In addition, previous studies reported that the inner retina during ischemia is more vulnerable to hypoxia, which is consistent with our previous research ${ }^{29,30}$. This reduction in inner retinal thickness may be associated with hypertensive retinal ischemia, such that chronic microvascular disorder is associated with slow inner retinal thinning. Similar to diabetic retinal neurodegeneration, it is possible that hypertensive changes can occur in patients without retinopathy. These were not observed using funduscopy, but there was still the possibility of microvascular damage. However, studies using OCT provide limited information on retinal capillary circulation.

The recently developed technique OCTA has no side effects from contrast agents, in contrast to FA, and has the advantage of being able to measure blood flow and the vascular density in SCP and DCP, separately. There have been many reports on the quantitative analysis of retinal vascular changes and ischemia in diabetic retinopathy, as 


\begin{tabular}{|l|l|l|l|l|l|l|l|}
\hline & $\begin{array}{l}\text { Control group } \\
(\mathbf{n}=\mathbf{9 0})\end{array}$ & $\begin{array}{l}\text { HTN group } \\
\mathbf{1}(\mathbf{n}=\mathbf{3 8})\end{array}$ & $\begin{array}{l}\text { HTN group } \\
\mathbf{2}(\mathbf{n}=\mathbf{4 0})\end{array}$ & $\mathbf{p}^{\text {-value* }}$ & $\mathbf{p}^{\text {-value }}$ & p-value $^{*}$ & $\mathbf{p}^{\text {-value }}$ \\
\hline Full area & $18.23 \pm 0.58$ & $18.02 \pm 0.77$ & $17.62 \pm 0.72$ & $<\mathbf{0 . 0 0 1}$ & 0.282 & $<\mathbf{0 . 0 0 1}$ & $\mathbf{0 . 0 2 6}$ \\
\hline Inner ring \\
\hline Average & $17.50 \pm 1.23$ & $17.12 \pm 1.80$ & $16.83 \pm 1.32$ & $\mathbf{0 . 0 3 6}$ & 0.489 & $\mathbf{0 . 0 3 9}$ & 1.000 \\
\hline Superior & $17.79 \pm 1.36$ & $17.58 \pm 1.85$ & $17.55 \pm 1.61$ & 0.632 & & & \\
\hline Nasal & $18.08 \pm 1.41$ & $17.61 \pm 2.15$ & $17.54 \pm 1.53$ & 0.136 & & & \\
\hline Inferior & $18.26 \pm 1.16$ & $18.06 \pm 1.30$ & $17.46 \pm 1.34$ & $\mathbf{0 . 0 0 4}$ & 1.000 & $\mathbf{0 . 0 0 3}$ & 0.103 \\
\hline Temporal & $16.32 \pm 2.41$ & $15.26 \pm 3.02$ & $15.13 \pm 2.54$ & $\mathbf{0 . 0 2 1}$ & 0.109 & $\mathbf{0 . 0 4 9}$ & 1.000 \\
\hline Outer ring & & & & & \\
\hline Average & $18.90 \pm 0.59$ & $18.74 \pm 0.63$ & $18.53 \pm 1.06$ & $\mathbf{0 . 0 3 5}$ & 0.872 & $\mathbf{0 . 0 3 1}$ & 0.613 \\
\hline Superior & $19.19 \pm 0.61$ & $19.15 \pm 0.68$ & $19.06 \pm 1.03$ & 0.622 & & & \\
\hline Nasal & $17.50 \pm 1.38$ & $17.47 \pm 1.07$ & $17.24 \pm 1.65$ & 0.595 & & & \\
\hline Inferior & $18.98 \pm 0.78$ & $18.91 \pm 0.86$ & $18.88 \pm 1.14$ & 0.808 & & & \\
\hline Temporal & $19.87 \pm 0.91$ & $19.43 \pm 1.31$ & $18.97 \pm 1.71$ & $\mathbf{0 . 0 0 1}$ & 0.193 & $\mathbf{0 . 0 0 1}$ & 0.318 \\
\hline
\end{tabular}

Table 3. Comparison of superficial peripapillary vessel density among groups. HTN = Hypertension. Values are presented as mean \pm standard deviation. ${ }^{*}$ The $p$-value was obtained using one-way analysis of variance. ${ }^{\dagger}$ The p-value was obtained using post hoc tests (Bonferroni) between the normal control group and HTN group 1. ¥The p-value was obtained using post hoc tests (Bonferroni) between the normal control group and HTN group 2. ${ }^{\S}$ The p-value was obtained using post hoc tests (Bonferroni) between HTN group 1 and group 2.

\begin{tabular}{|l|l|l|l|l|l|l|l|l|}
\hline & $\begin{array}{l}\text { Control group } \\
(\mathbf{n = 9 0 )}\end{array}$ & $\begin{array}{l}\text { HTN group 1 } \\
(\mathbf{n = 3 8})\end{array}$ & $\begin{array}{l}\text { HTN group 2 } \\
(\mathbf{n = 4 0 )}\end{array}$ & p-value $^{*}$ & p-value $^{\dagger}$ & p-value $^{*}$ & p-value $^{\mathfrak{s}}$ \\
\hline Full area & $0.465 \pm 0.013$ & $0.460 \pm 0.020$ & $0.449 \pm 0.020$ & $<\mathbf{0 . 0 0 1}$ & 0.274 & $<\mathbf{0 . 0 0 1}$ & $\mathbf{0 . 0 1 9}$ \\
\hline Inner ring \\
\hline Average & $0.459 \pm 0.032$ & $0.450 \pm 0.047$ & $0.441 \pm 0.039$ & $\mathbf{0 . 0 4 9}$ & 0.703 & $\mathbf{0 . 0 4 9}$ & 0.934 \\
\hline Superior & $0.472 \pm 0.041$ & $0.466 \pm 0.049$ & $0.468 \pm 0.048$ & 0.745 & & & \\
\hline Nasal & $0.482 \pm 0.033$ & $0.468 \pm 0.061$ & $0.467 \pm 0.047$ & 0.118 & & & \\
\hline Inferior & $0.486 \pm 0.030$ & $0.485 \pm 0.035$ & $0.470 \pm 0.041$ & $\mathbf{0 . 0 4 9}$ & 1.000 & 0.056 & 0.159 \\
\hline Temporal & $0.393 \pm 0.060$ & $0.375 \pm 0.077$ & $0.358 \pm 0.071$ & $\mathbf{0 . 0 2 5}$ & 0.794 & $\mathbf{0 . 0 2 1}$ & 0.548 \\
\hline Outer ring & & & & & & \\
\hline Average & $0.479 \pm 0.014$ & $0.475 \pm 0.020$ & $0.465 \pm 0.024$ & $<\mathbf{0 . 0 0 1}$ & 0.843 & $<\mathbf{0 . 0 0 1}$ & $\mathbf{0 . 0 3 8}$ \\
\hline Superior & $0.496 \pm 0.016$ & $0.493 \pm 0.017$ & $0.492 \pm 0.017$ & 0.310 & & & \\
\hline Nasal & $0.451 \pm 0.032$ & $0.449 \pm 0.043$ & $0.432 \pm 0.047$ & $\mathbf{0 . 0 4 0}$ & 1.000 & $\mathbf{0 . 0 4 0}$ & 0.178 \\
\hline Inferior & $0.488 \pm 0.019$ & $0.481 \pm 0.024$ & $0.476 \pm 0.031$ & $\mathbf{0 . 0 3 6}$ & 0.504 & $\mathbf{0 . 0 3 8}$ & 1.000 \\
\hline Temporal & $0.481 \pm 0.020$ & $0.473 \pm 0.024$ & $0.459 \pm 0.048$ & $\mathbf{0 . 0 0 1}$ & 0.443 & $<\mathbf{0 . 0 0 1}$ & 0.135 \\
\hline
\end{tabular}

Table 4. Comparison of superficial peripapillary perfusion density among groups. HTN = Hypertension. Values are presented as mean \pm standard deviation. ${ }^{*}$ The $p$-value was obtained using one-way analysis of variance. ${ }^{\dagger}$ The $\mathrm{p}$-value was obtained using post hoc tests (Bonferroni) between the normal control group and HTN group 1. ${ }^{\ddagger}$ The $\mathrm{p}$-value was obtained using post hoc tests (Bonferroni) between the normal control group and HTN group 2. ${ }^{\$}$ The p-value was obtained using post hoc tests (Bonferroni) between HTN group 1 and group 2 .

well as on retinal venous occlusion and glaucoma. Recent studies with OCTA have shown decreased foveal microvascular parameters, increased foveal avascular zone areas in patients with chronic HTN, and relived HTNR. Because of the association between the microvasculature and inner retinal layer thickness, inner retinal thinning may be associated with retinal microcirculation ${ }^{5,6}$. In addition, patients with poorly controlled blood pressure showed a greater decrease in the retinal capillary density compared with patients whose blood pressure is well controlled ${ }^{31}$.

To our knowledge, there has been no previous study on changes to the peripapillary microvasculature according to HTN using OCTA. We found that the average peripapillary VD and PD of the inner ring, outer ring, and full area were different among the three groups. The peripapillary VD and PD of HTN Group 2 were lower than those of normal controls. We evaluated associations between OCT and peripapillary OCTA parameters in HTN patients. The peripapillary VD and PD showed a correlation with the GC-IPL and RNFL thicknesses. The decreased GC-IPL and peripapillary RNFL thickness might be associated with decreased peripapillary microvascular circulation. In the present study, the HTN duration did not correlate with the GC-IPL and RNFL thicknesses, but was correlated with the peripapillary VD and PD. It is presumed that the peripapillary microvasculature was more sensitive to the duration of HTN than the inner retinal thickness. 

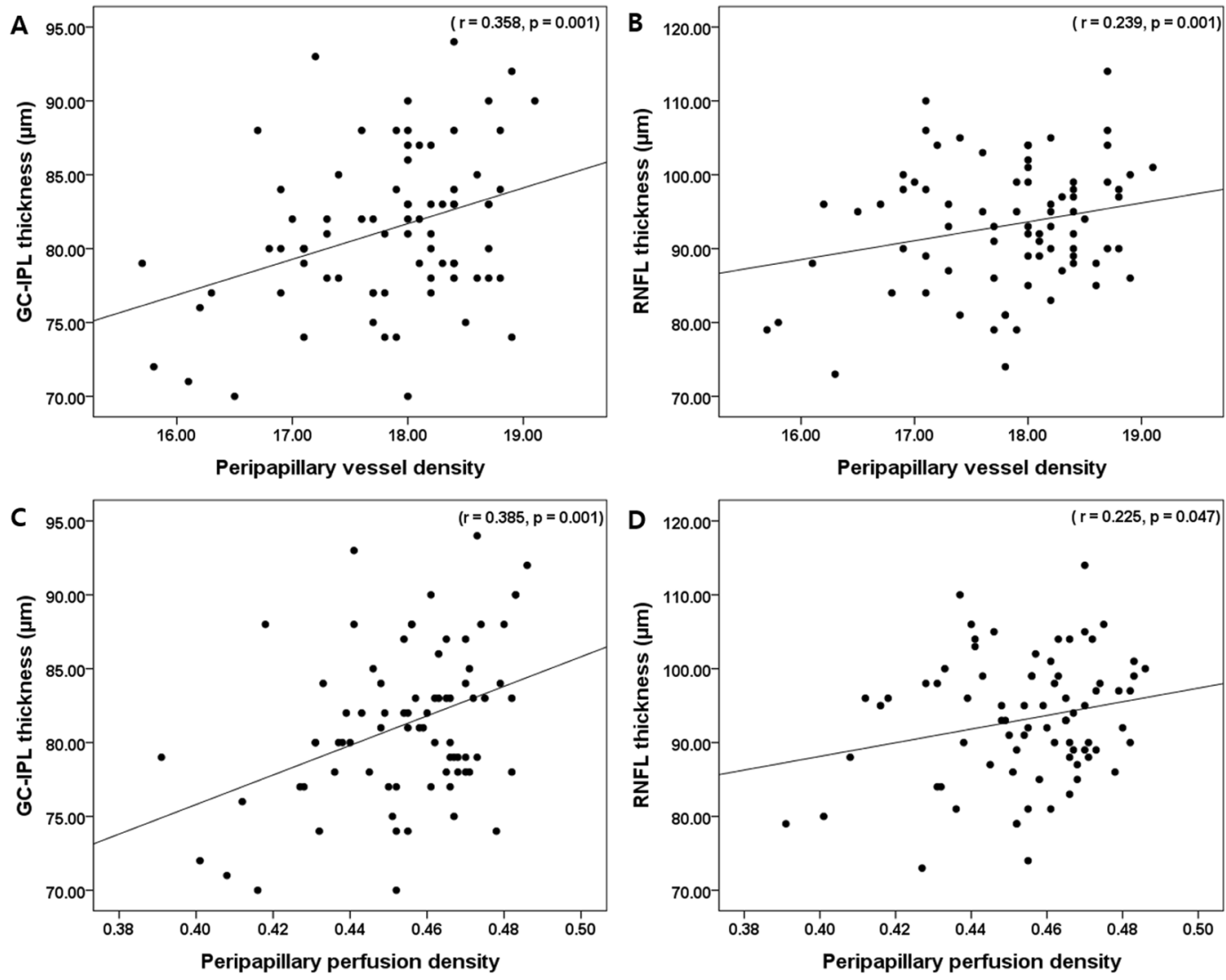

Figure 2. Scatter plots of association with peripapillary OCTA parameters with OCT parameters. Peripapillary vessel density (VD) and perfusion density (PD) were significantly correlated with average ganglion cellinner plexiform layer (GC-IPL) and retinal nerve fiber layer (RNFL) thickness in HTN patients. Correlation coefficients (r) and $p$-values are shown.

This study has some limitations. First, in this retrospective, cross-sectional study, it was difficult to identify the occurrence of previous hypertensive events. In general, if there is no acute rise in blood pressure, visual symptoms do not occur, and ophthalmic examinations are often not performed. We excluded patients in HTNR with cotton wool spots, retinal hemorrhage, and severe arteriolar narrowing, thereby minimizing the effect of these on peripapillary OCTA parameters. Second, anti-hypertensive medications and kidney functioning (low estimated glomerular filtration rate levels) could affect the retinal microcirculation ${ }^{32,33}$. Because of the cross-sectional study, we could not investigate these parameters in detail. Previous studies ${ }^{32,33}$ used scanning laser Doppler Flowmetry and fundus photography, so additional studies with OCTA may be needed. Third, a further longitudinal study of temporal sequencing between decreased GC-IPL and RNFL thicknesses and retinal microvasculature should be conducted, and the DCP and choroid investigated, as they may be affected by HTN. Finally, we only analyzed SCP, not DCP and choriocapillaries. However, previous studies have shown that projection artifacts may affect quantitative analysis of DCP and choriocapillaries, and Enders et al. ${ }^{34}$ reported that $75 \%$ of deep retina images were shown projection artifacts. Further studies on the changes of DCP and choriocapillaries using projection-resolved OCTA will be needed.

Our study excluded systemic diseases other than HTN. It is important to investigate the only effects of HTN on peripapillary microvasculature. In addition, we studied changes in peripapillary optic nerve head perfusion in HTN patients for the first time. Overall, the strength of this study was its characterization of the relationship between peripapillary microvasculature and the RNFL and GC-IPL thicknesses.

In conclusion, this study showed that the peripapillary VD and PD were lower in HTN patients with a duration of $\geq 10$ years, and the peripapillary VD and PD showed a correlation with RNFL and GC-IPL thicknesses. Based on the findings in this study, the effects of HTN duration should be taken into account when evaluating the peripapillary microvasculature using OCTA.

\section{Data availability}

Data supporting the findings of the current study are available from the corresponding author on reasonable request.

Received: 28 October 2019; Accepted: 1 April 2020;

Published online: 16 April 2020 


\section{References}

1. Vital signs. prevalence, treatment, and control of hypertension-United States, 1999-2002 and 2005-2008. MMWR. Morbidity and Mortality Weekly Report 60, 103-108 (2011).

2. Chobanian, A. V. et al. The Seventh Report of the Joint National Committee on Prevention, Detection, Evaluation, and Treatment of High Blood Pressure. The JNC 7 Report. JAMA 289, 2560-2571, https://doi.org/10.1001/jama.289.19.2560 (2003).

3. Lee, H. M., Lee, W. H., Kim, K. N., Jo, Y. J. \& Kim, J. Y. Changes in thickness of central macula and retinal nerve fibre layer in severe hypertensive retinopathy: a 1-year longitudinal study. Acta Ophthalmologica 96, e386-e392 (2018).

4. Lee, S. H., Lee, W. H., Lim, H. B., Jo, Y. J. \& Kim, J. Y. Thicknesses of Central Macular, Retinal Nerve Fiber, and Ganglion Cell Inner Plexiform Layers in Patients with Hypertension. Retina (Philadelphia, Pa.) (2018).

5. Lee, W. H. et al. Retinal Microvascular Change in Hypertension as measured by Optical Coherence Tomography Angiography. Scientific Reports 9, 156 (2019).

6. Lim, H. B. et al. Changes in Ganglion Cell-Inner Plexiform Layer Thickness and Retinal Microvasculature in Hypertension: An Optical Coherence Tomography Angiography Study. American Journal of Ophthalmology 199, 167-176 (2019).

7. Lee, H.-Y. \& Park, J. B. The Korean Society of Hypertension Guidelines for the Management of Hypertension in 2013: its essentials and key points. Pulse 3, 21-28 (2015).

8. Faul, F., Erdfelder, E., Buchner, A. \& Lang, A. G. Statistical power analyses using G*Power 3.1: tests for correlation and regression analyses. Behavior Research Methods 41, 1149-1160, https://doi.org/10.3758/brm.41.4.1149 (2009).

9. Wong, T. Y. et al. Retinal microvascular abnormalities and 10-year cardiovascular mortality: a population-based case-control study. Ophthalmology 110, 933-940, https://doi.org/10.1016/s0161-6420(03)00084-8 (2003).

10. Wong, T. Y. et al. Retinopathy and risk of congestive heart failure. Jama 293, 63-69, https://doi.org/10.1001/jama.293.1.63 (2005).

11. Yatsuya, H. et al. Retinal microvascular abnormalities and risk of lacunar stroke: Atherosclerosis Risk in Communities Study. Stroke 41, 1349-1355, https://doi.org/10.1161/strokeaha.110.580837 (2010).

12. Wong, T. Y. et al. Retinal microvascular abnormalities and renal dysfunction: the atherosclerosis risk in communities study. Journal of the American Society of Nephrology: JASN 15, 2469-2476, https://doi.org/10.1097/01.asn.0000136133.28194.e4 (2004).

13. Yip, W. et al. Retinal Vascular Imaging Markers and Incident Chronic Kidney Disease: A Prospective Cohort Study. Scientific Reports 7, 9374, https://doi.org/10.1038/s41598-017-09204-2 (2017).

14. Kassab, E., McFarlane, S. I. \& Sower, J. R. Vascular complications in diabetes and their prevention. Vascular Medicine (London, England) 6, 249-255 (2001).

15. Group, E. D. C.-C. S. Risk factors for branch retinal vein occlusion. American Journal of Ophthalmology 116, 286-296 (1993).

16. Hayreh, S. S., Podhajsky, P. A. \& Zimmerman, M. B. Retinal artery occlusion: associated systemic and ophthalmic abnormalities. Ophthalmology 116, 1928-1936 (2009).

17. Hayreh, S. S. Ischemic optic neuropathy. Progress in Retinal and Eye Research 28, 34-62, https://doi.org/10.1016/j. preteyeres.2008.11.002 (2009).

18. Leighton, D. \& Phillips, C. Systemic blood pressure in open-angle glaucoma, low tension glaucoma, and the normal eye. The British Journal of Ophthalmology 56, 447 (1972).

19. Bonomi, L. et al. Vascular risk factors for primary open angle glaucoma: the Egna-Neumarkt Study. Ophthalmology 107, 1287-1293 (2000).

20. Maguire, M. G. et al. Risk factors for choroidal neovascularization in the second eye of patients with juxtafoveal or subfoveal choroidal neovascularization secondary to age-related macular degeneration. Archives of Ophthalmology 115, 741-747 (1997).

21. Chobanian, A. V. et al. The seventh report of the joint national committee on prevention, detection, evaluation, and treatment of high blood pressure: the JNC 7 report. Jama 289, 2560-2571 (2003).

22. McGeechan, K. et al. Prediction of incident stroke events based on retinal vessel caliber: a systematic review and individualparticipant meta-analysis. American Journal of Epidemiology 170, 1323-1332, https://doi.org/10.1093/aje/kwp306 (2009).

23. Hayreh, S. S. Hypertensive retinopathy. Introduction. Ophthalmologica. Journal International D'ophtalmologie. International Journal of Ophthalmology. Zeitschrift fur Augenheilkunde 198, 173-177 (1989).

24. Wong, T. Y., Klein, R., Klein, B. E., Meuer, S. M. \& Hubbard, L. D. Retinal vessel diameters and their associations with age and blood pressure. Investigative Ophthalmology \& Visual Science 44, 4644-4650 (2003).

25. Leung, H. et al. Impact of current and past blood pressure on retinal arteriolar diameter in an older population. Journal of Hypertension 22, 1543-1549 (2004).

26. Hubbard, L. D. et al. Methods for evaluation of retinal microvascular abnormalities associated with hypertension/sclerosis in the Atherosclerosis Risk in Communities Study. Ophthalmology 106, 2269-2280 (1999).

27. Akay, F. et al. Retinal structural changes in systemic arterial hypertension: an OCT study. European Journal of Ophthalmology 26, 436-441, https://doi.org/10.5301/ejo.5000740 (2016).

28. Hayreh, S. S. \& Jonas, J. B. Ophthalmoscopic detectability of the parafoveal annular reflex in the evaluation of the optic nerve: an experimental study in rhesus monkeys. Ophthalmology 107, 1009-1014 (2000).

29. Ebneter, A., Agca, C., Dysli, C. \& Zinkernagel, M. S. Investigation of retinal morphology alterations using spectral domain optical coherence tomography in a mouse model of retinal branch and central retinal vein occlusion. PloS one 10, e0119046, https://doi. org/10.1371/journal.pone.0119046(2015).

30. Yu, D. Y., Cringle, S. J. \& Su, E. N. Intraretinal oxygen distribution in the monkey retina and the response to systemic hyperoxia. Investigative Ophthalmology \& Visual Science 46, 4728-4733, https://doi.org/10.1167/iovs.05-0694 (2005).

31. Chua, J. et al. Impact of hypertension on retinal capillary microvasculature using optical coherence tomographic angiography. Journal of Hypertension 37, 572-580, https://doi.org/10.1097/hjh.0000000000001916 (2019).

32. Hughes, A. D. et al. Effect of antihypertensive treatment on retinal microvascular changes in hypertension. Journal of Hypertension 26, 1703-1707 (2008)

33. Bosch, A. et al. Retinal capillary and arteriolar changes in patients with chronic kidney disease. Microvascular Research 118, 121-127 (2018).

34. Enders, C. et al. Quantity and quality of image artifacts in optical coherence tomography angiography. PloS one 14, e0210505, https://doi.org/10.1371/journal.pone.0210505 (2019).

\section{Author contributions}

Design and conduct of the study (Y.I.S., Y.J.J., J.Y.K.); Collection of data (Y.I.S., H.B.L., J.Y.K.); Analysis and interpretationof data (Y.I.S., W.-H.L., C.K.R., Y.J.J., J.Y.K.); Writing the article (Y.I.S., H.B.L., J.Y.K.); Critical revision of the article (K.-Y.N., Y.J.J., J.Y.K.); Final approval ofthe article (Y.I.S., K.-Y.N., W.-H.L., C.K.R., H.B.L., Y.J.J., J.Y.K.).

\section{Competing interests}

The authors declare no competing interests. 


\section{Additional information}

Supplementary information is available for this paper at https://doi.org/10.1038/s41598-020-63603-6.

Correspondence and requests for materials should be addressed to J.-Y.K.

Reprints and permissions information is available at www.nature.com/reprints.

Publisher's note Springer Nature remains neutral with regard to jurisdictional claims in published maps and institutional affiliations.

(c) (i) Open Access This article is licensed under a Creative Commons Attribution 4.0 International License, which permits use, sharing, adaptation, distribution and reproduction in any medium or format, as long as you give appropriate credit to the original author(s) and the source, provide a link to the Creative Commons license, and indicate if changes were made. The images or other third party material in this article are included in the article's Creative Commons license, unless indicated otherwise in a credit line to the material. If material is not included in the article's Creative Commons license and your intended use is not permitted by statutory regulation or exceeds the permitted use, you will need to obtain permission directly from the copyright holder. To view a copy of this license, visit http://creativecommons.org/licenses/by/4.0/.

(c) The Author(s) 2020 